\title{
Brain Aminergic Deficiency in Absence Epileptic Rats: Dependency on Seizure Severity and Their Functional Coupling at Rest
}

\author{
I. S. Midzyanovskaya ${ }^{1,2 *}$, A. B. Shatskova ${ }^{2}$, E. MacDonald ${ }^{1}$, G. Van Luijtelaar ${ }^{3}$, L. Tuomisto ${ }^{1}$ \\ ${ }^{1}$ School of Pharmacy (Pharmacology and Toxicology), University of Eastern Finland, Kuopio, Finland \\ ${ }^{2}$ Institution of Higher Nervous Activity and Neurophysiology, Russian Academy of Science, Moscow, Russia \\ ${ }^{3}$ Donders Centre for Cognition, Radboud University, Nijmegen, Netherlands \\ Email: *miinn@yandex.ru
}

How to cite this paper: Midzyanovskaya, I.S., Shatskova, A.B., MacDonald, E., Van Luijtelaar, G. and Tuomisto, L. (2020) Brain Aminergic Deficiency in Absence Epileptic Rats: Dependency on Seizure Severity and Their Functional Coupling at Rest. Journal of Behavioral and Brain Science, 10, 29-45. https://doi.org/10.4236/jbbs.2020.101003

Received: November 28, 2019

Accepted: January 7, 2020

Published: January 10, 2020

Copyright $\odot 2020$ by author(s) and Scientific Research Publishing Inc. This work is licensed under the Creative Commons Attribution International License (CC BY 4.0).

http://creativecommons.org/licenses/by/4.0/

\begin{abstract}
Brain aminergic tone was examined in rats with different genetically determined generalized epilepsies and in normal rats. Our previously published database was analyzed to examine if there were functional correlations between brain biogenic amines and their metabolic rates within particular cerebral regions. Rats with genetically determined absence (AbS) and/or audiogenic seizures (AGS) served as models for human generalized non-convulsive and convulsive epilepsies. Tissue concentrations of 5-HT, dopamine (DA) and their main metabolites, as well as tryptophan (TRT), histamine (HA) and noradrenaline (NA) were assayed in different brain regions and analyzed for within-regions correlations in non-stressed conditions. It was found that $\mathrm{AbS}$ rats had higher indices of DA-ergic metabolism than non-epileptic controls, but the severity of AbS correlated with a widespread dopaminergic deficiency, as expressed in lowered indices of DA metabolism. AGS exerted no major effects on tissue neurochemistry. Brain DA-ergic and 5HT-ergic metabolic indices (measured as the ratio of the metabolite to the parent transmitter) were closely linked in striatum and pons-medulla of AbS rats. Remarkably, the tissue content of HA in pons-medulla of AbS rats displayed correlations with metabolic ratios of $5 \mathrm{HT}$ and DA, which was not seen in normal rats. It is hypothesized that enhanced metabolism of DA and 5HT are at least partial compensatory antiepileptic mechanisms in the brain, and a disturbance of this compensation leads to an aggravation of absence seizures.
\end{abstract}

\section{Keywords}

Absence Epilepsy, Rat, Animal Model, Brain Monoamines, Histamine 


\section{Introduction}

The brain aminergic system performs a multitude of functions, being implicated in the control of arousal, mood, cognition, locomotion, etc. This system can modulate the excitability of many neuronal networks throughout the brain [1] [2] [3] [4], and thus influence the paroxysmal threshold and thus the occurrence of epileptic seizures [3] [5] [6] [7]. Recently, a method of correlative analysis was applied to inter-individual differences in brain regional aminergic indices e.g. to evaluate interactions between the dopaminergic and serotoninergic systems within and between cerebral regions [8] [9] [10]. In the present study, we applied this approach to our published datasets [11], in order to reveal the interactions between regional histamine (HA)-, dopamine (DA)-, norepinephrine (NA)- and serotonine $(5 \mathrm{HT})$-ergic systems in normal and epileptic brains utilizing our previous separate datasets [11] [12] [13].

A deficiency of the brain aminergic neurotransmission is known to facilitate both convulsive and non-convulsive seizures in experimental animals, whereas an experimentally-induced increase of aminergic activity exerts clear anti-epileptic effects in rodents [14]-[21]. A delayed increase in the release of catecholamines, which follows the induction of electroencephalographic and behavioral seizures in rats, is thought to be a compensatory action [19]. Another issue that it is still not totally understood is whether the brain aminergic systems undergo functional changes during spontaneous epileptogenesis. This is particularly unclear in the case of the non-convulsive epilepsies. We have investigated this by examining a genetic animal model of childhood absence epilepsy-rats of the WAG/Rij rat strain [21] [22] [23]. Virtually all adult rats of this strain develop the EEG hallmarks of absence seizures (AbS)-widely generalized spike-wave discharges (SWDs), concomitant with behavioral arrest and mild orofacial jerks. The inter-individual differences in the propensity towards absence seizures in WAG/Rij rats [23] are likely attributable to some unknown internal modulatory mechanisms [24]. We hypothesized that an inter-individual difference in the severity of absence seizures would mimic the putative within-individual changes in time; i.e. a comparison between subjects with low to high epileptic scores could provide valuable information on the mechanisms of aggravation of seizures in epileptic individuals. Therefore, we compared aminergic indices in different brain regions of WAG/Rij rats differing in their severity of AbS and analyzed whether this displayed functional correlations with the different biogenic amines. AGS-susceptible individuals of the Wistar and WAG/Rij rat strains [24] [25] were also examined in the original studies [11] [12] [13]. It was shown that effects of AGS susceptibility on brain amines are minimal at rest but appear to be more significant in stressed animals [11] [12] [13]. The effects of mild but recurrent $\mathrm{AbS}$ were prominent and widespread both at rest and in the stress response [11] [12] [13]. In the present study, we concentrated on the functional coupling of biogenic amines in the brain of non-stressed animals.

The tissue contents of the neurotransmitter in the rats with absence and/or 
audiogenic epilepsy have been already published by our group [11] [12] [13]. However, the tissue content itself represents mainly the neurotransmitter stored at high concentrations in different vesicular compartments inside neurons [26] [27] [28] [29]. Brain aminergic systems represent a potential target for a complex therapy of epilepsy and comorbid disorders, such as anxiety, depression and others [3] [4]. To develop aminergic strategies of treatment, it is needed to better understand functional interaction of these neuromodulatory systems. It is quite possible that the epileptic brain would have a seizure-type specific pattern for such interaction. The assessment of functional coupling between the brain aminergic systems should also involve metabolic rates [7] [8] [9], which are reflected in the ratios of metabolite(s) to parent neurotransmitter/precursor, measured simultaneously in the same brain regions [7] [8] [9] [10]. Therefore, we applied the correlative approach, introduced by De Deurwaerdère and colleagues [7] [8] [9], to our dataset on brain biogenic amines in rats with genetically determined generalized epilepsies [11] [12] [13]. The present analysis renders it possible to asses if the activity of aminergic systems might act as antiepileptic compensatory mechanisms.

\section{Methods}

Animals. A total of 20 rats were examined. Male rats of the WAG/Rij $(\mathrm{N}=10)$ and Wistar $(\mathrm{N}=10)$ strains, 5 - 6 months old, were used in the experiment. Audiogenic susceptibility was pre-examined by two sound tests, separated by $7-9$ days. The sound test was the same as that described earlier [24] [25]: complex sound with a frequency range of $13-85 \mathrm{kHz}$, maximum in $20-40 \mathrm{kHz}$ and mean intensity $50-60 \mathrm{~dB}$, was automatically presented for 90 seconds in an experimental chamber $(90 \times 90 \mathrm{~cm})$. The rats involved in the present study displayed mild seizures, wild running (score 1 ) or wild running followed by jumping (score 2). A failure to experience an AGS was scored as 0 [24]. The final score that was assigned to an animal was the average of the scores in the two successive tests. Five rats of each strain which exhibited AGS in two successive tests were selected; as well as five other rats per strain, which did not show any sign of AGS. Thus, four experimental groups were formed: Wistar rats with audiogenic seizures (Wistar-AGS), Wistar control rats served as non-epileptic controls (Wistar), WAG/Rij rats without AGS (WAG/Rij), and WAG/Rij rats with audiogenic seizures (WAG/Rij-AGS). The latter group had a double or mixed epileptic pathology.

All rats were housed at 3 - 5 animals per cage, under natural light-dark conditions (about 10 hours of light in the daytime) and with ad libitum access to standard pellet food and water. All experiments were performed in accordance with the institutional and national guidelines of animal care. All efforts were made to reduce suffering of the animals and to minimize the number of experimental subjects involved.

Surgery: Ten days after the second test for AGS susceptibility, the epidural cortical electrodes (stainless steel screws) were implanted in each rat under 
chloral hydrate narcosis (4\% solution, $10 \mathrm{ml} / \mathrm{kg}$ i.p.) and local anesthesia (procaine $2 \%$ solution).

ECoG registrations. After a two-week post-surgery recovery period, the ECoG's of the rats were recorded in two sessions of 45 - 60 minutes, conducted between 16.00 and 19.00 p.m. Criteria of SWDs were as previously published [30]. Individual indices of spike-wave activity (SWI) were calculated as a mean percentage of the time occupied by SWDs.

Decapitation and dissection: During the 3 - 4 weeks before the final experiment, the animals were occasionally brought to the room where they would be sacrificed, so that they would become habituated to the environment: each rat had 4 - 5 habituation sessions, randomly scheduled within this period. During this period, also behavioural tests were performed in the animals (the open field and light-dark choice tests). The results of the behavioural experiments were reported elsewhere [31].

The rats were decapitated 10 to 14 days after the last ECoG-session in the same room to which they had been habituated to the experimental conditions; the order of decapitation was counterbalanced. The brains were dissected from the skull, vascular tissue was carefully removed and brain regions were dissected on ice. Tissue samples of the frontal and parieto-occipital cortices were cut with a thin metal wire. Next, the amygdala, striatum, thalamus, hypothalamus and pons-medulla oblongata were dissected according to [30]. The samples were individually placed into plastic tubes and frozen on top of dry ice. Subsequently, the samples were stored at $-70^{\circ} \mathrm{C}$ until HPLC assay.

All thawed samples were first homogenized in ice-cold $0.4 \mathrm{M}$ perchloric acid and the homogenates were centrifuged at $100,000 \mathrm{~g}$ for 45 minutes at $4^{\circ} \mathrm{C}$; supernatants were frozen until the assay.

Monoamine assay: NA, DA and its main metabolites homovanillic acid (HVA) and 3,4-dihydroxyphenylacetic acid (DOPAC) were assayed from brain samples by HPLC coupled with an electrochemical (coulometric) detector [32]. The concentrations of tryptophan (TRT) as well as serotonin (5-hydroxytrptamine, 5-HT) and its metabolite, 5-hydroxyindoleacetic acid (5-HIAA) were also assayed electrochemically in the same HPLC run as the catecholamines.

Histamine assay: Supernatants were used for HPLC assay, with post-column derivatization and fluorescence detection, as described by Yamatodani et al. [33].

Statistics. The datasets for each brain region were processed by ANOVA general linear model (GLM) with "strain" and "AGS" as the between-subject factors, SWI was taken as a continuous predictor. Single missing values were replaced with the corresponding group means, to allow a complete statistical analysis of other variables. This procedure does not affect the group means and between-group differences.

The rats of the same strain were pooled into one group if there was no significant difference nor a tendency to differ between AGS-prone animals and AGS-resistant rats. Those parameters, which demonstrated a significant difference between AGS-susceptible and AGS-resistant groups, were excluded from the subsequent 
correlative analysis. The correlations between neurochemical values measured were estimated by the Spearman method of rank order correlation. Fisher exact $2 \times 2$ test was used in order to determine whether the numbers of regional correlations differed significantly between the two strains.

All data are given as mean \pm SEM, $p=0.05$ was accepted as a minimal level of significance, $\mathrm{p}<0.1$ was considered as a tendency.

\section{Results}

\subsection{Paroxysmal Activity}

AGS was scored as described earlier [24] [25]. Mean AGS scores were rather similar for Wistar-AGS and WAG/Rij-AGS rats (1.4 and 1.1, respectively). The AbS was scored as SWI indices $(3.3 \pm 0.77$ and $3.3 \pm 1.08$ for WAG/Rij and WAG/Rij-AGS rats, respectively). No paroxysmal activity was registered in ECoG of Wistar rats.

\subsection{AGS}

No specific effect was seen in the AGS-susceptibility when the animals were at rest on the monoaminergic parameters assayed in the present study (Table 1). Tendencies towards an $\mathrm{AGS}^{\star}$ strain interaction were detected in frontal cortex for NA, in thalamus for HA, in hypothalamus for HVA and HVA/DA ratio, in pons-medulla for 5HT/TRT ratio. These tendencies have been reported earlier [11] [12] [13]. The regional parameters mentioned above were excluded from the correlative analysis.

The effect of AbS was dependent on the strain of rats being examined, since only WAG/Rij rats were susceptible to AbS in our study.

Frontal cortex: A significant strain effect was found for all metabolic ratios of $\mathrm{DA}$, they were elevated, or tended towards an elevation in WAG/Rij groups as compared to Wistar rats $(\{\mathrm{F}(1,19)=4.40, \mathrm{p}<0.05\},\{\mathrm{F}(1,19)=4.63, \mathrm{p}<0.05\}$, and $\{\mathrm{F}(1,19)=4.57, \mathrm{p}<0.05$ for DOPAC/DA, $(\mathrm{DOPAC}+\mathrm{HVA}) / \mathrm{DA}$ and NA/DA, respectively\}, $\mathrm{p}=0.06$ for HVA/DA.

Parietal cortex: Metabolic indices of dopamine were elevated (strain effect, $\{\mathrm{F}(1,18)=5.04, \mathrm{p}<0.05\}$ for $(\mathrm{DOPAC}+\mathrm{HVA}) / \mathrm{DA},(\{\mathrm{F}(1,18)=4.69, \mathrm{p}<0.05$ for NA/DA in WAG/Rij groups, as well as that of serotonin (strain effect $\{\mathrm{F}(1,18)=6.81, \mathrm{p}<0.05\}$ for $5 \mathrm{HIAA} / 5 \mathrm{HT})$. Tissue HA tended to be lower in WAG/Rij rats $\{$ strain effect, $\mathrm{F}(1,18)=3.99, \mathrm{p}=0.06\}$.

Striatum: Tissue HA was lower in WAG/Rij groups \{strain effect $F(1,19)=$ $19.78, \mathrm{p}<0.001\}$ compared to Wistar groups.

Hypothalamus: Tissue HA and TRT was higher in WAG/Rij rats compared to Wistar rats (strain effect $\{\mathrm{F}(1,19)=7.42, \mathrm{p}<0.05\}$ and $\{\mathrm{F}(1,19)=4.42, \mathrm{p}<0.05\}$, respectively).

Amygdala: TRT was elevated in WAG/Rij rats as compared to Wistar ones $\{$ strain effect $\mathrm{F}(1,19)=5.37, \mathrm{p}<0,05\}$, as well as NA/DA ratio $\{\mathrm{F}(1,19)=4.47, \mathrm{p}$ $<0.05\}$. 
Table 1. Overview of tissue content of monoamines and some metabolites in cortical (a) and subcortical (b) regions of Wistar and WAG/Rij rats. The content of each monoamine and metabolite is expressed in ng/mg \pm SEM. The metabolic ratios for DA or 5-HT systems have been reported as the ratios of the tissue content of DOPAC, HVA or (DOPAC + HVA) to and DA; or 5HT to TRT, 5-HIAA to 5-HT, respectively. Strain difference and/or effect of AGS are highlighted and signed below the corresponded columns.

(a)

\begin{tabular}{|c|c|c|c|c|c|c|c|c|}
\hline frontal ctx & NA & $\mathrm{DA}$ & DOPAC & HVA & $5 \mathrm{HT}$ & 5HIAA & TRT & HA \\
\hline Wistar & $1.50 \pm 0.18$ & $0.43 \pm 0.23$ & $0.38 \pm 0.15$ & $0.15 \pm 0.03$ & $1.81 \pm 0.21$ & $3.03 \pm 0.78$ & $2.48 \pm 0.37$ & $0.29 \pm 0.06$ \\
\hline WAG/Rij & $2.15 \pm 0.13$ & $0.64 \pm 0.32$ & $0.46 \pm 0.15$ & $0.20 \pm 0.07$ & $2.01 \pm 0.39$ & $4.16 \pm 0.95$ & $2.80 \pm 0.49$ & $0.22 \pm 0.04$ \\
\hline Wistar-AGS & $2.09 \pm 0.21$ & $0.83 \pm 0.31$ & $0.60 \pm 0.19$ & $0.19 \pm 0.05$ & $2.40 \pm 0.33$ & $3.78 \pm 0.95$ & $2.95 \pm 0.17$ & $0.20 \pm 0.02$ \\
\hline WAG/Rij-AGS & $1.99 \pm 0.21$ & $\mathbf{0 . 4 4} \pm 0.15$ & $0.38 \pm 0.07$ & $0.14 \pm 0.03$ & $2.07 \pm 0.34$ & $3.29 \pm 0.79$ & $2.57 \pm 0.39$ & $0.22 \pm 0.07$ \\
\hline parietal ctx & $\begin{array}{c}\text { strain* }^{*} \\
\text { AGS-tend }\end{array}$ & & & & & & $\operatorname{strain}^{*} A G S$ & \\
\hline Wistar & $0.47 \pm 0.19$ & $0.06 \pm 0.03$ & $0.03 \pm 0.01$ & $0.01 \pm 0.01$ & $0.39 \pm 0.23$ & $1.67 \pm 1.42$ & $3.08 \pm 0.15$ & $0.25 \pm 0.02$ \\
\hline WAG/Rij & $0.65 \pm 0.35$ & $0.11 \pm 0.08$ & $0.03 \pm 0.02$ & $0.02 \pm 0.01$ & $0.41 \pm 0.30$ & $1.95 \pm 1.68$ & $3.09 \pm 0.12$ & $0.21 \pm 0.02$ \\
\hline Wistar-AGS & $0.44 \pm 0.24$ & $0.10 \pm 0.06$ & $0.04 \pm 0.03$ & $0.02 \pm 0.01$ & $0.45 \pm 0.29$ & $1.53 \pm 1.20$ & $3.14 \pm 0.30$ & $0.28 \pm 0.02$ \\
\hline $\begin{array}{c}\text { WAG/Rij-AGS } \\
\text { amygdala }\end{array}$ & $0.57 \pm 0.15$ & $0.14 \pm 0.06$ & $0.01 \pm 0.01$ & $0.02 \pm 0.01$ & $0.29 \pm 0.12$ & $1.43 \pm 1.14$ & $3.48 \pm 0.26$ & $\begin{array}{c}0.19 \pm 0.02 \\
\text { strain }^{*}-\text { tend }\end{array}$ \\
\hline Wistar & $4.18 \pm 0.58$ & $2.28 \pm 0.79$ & $0.37 \pm 0.05$ & $0.34 \pm 0.04$ & $3.19 \pm 0.51$ & $3.70 \pm 0.34$ & $3.44 \pm 0.10$ & $0.30 \pm 0.05$ \\
\hline WAG/Rij & $5.49 \pm 0.74$ & $1.30 \pm 0.27$ & $0.30 \pm 0.04$ & $0.34 \pm 0.10$ & $4.23 \pm 0.54$ & $4.65 \pm 0.28$ & $4.27 \pm 0.24$ & $0.34 \pm 0.02$ \\
\hline Wistar-AGS & $2.99 \pm 0.83$ & $1.57 \pm 0.33$ & $0.37 \pm 0.11$ & $0.46 \pm 0.07$ & $3.36 \pm 0.74$ & $3.46 \pm 0.61$ & $3.45 \pm 0.44$ & $0.29 \pm 0.05$ \\
\hline WAG/Rij-AGS & $\begin{array}{c}5.59 \pm 0.77 \\
\text { strain }^{*}\end{array}$ & $2.35 \pm 0.75$ & $0.36 \pm 0.18$ & $0.46 \pm 0.10$ & $\begin{array}{c}3.92 \pm 0.62 \\
\text { strain }^{*}\end{array}$ & $\begin{array}{c}4.68 \pm 0.19 \\
\text { strain }^{*}\end{array}$ & $\begin{array}{c}4.27 \pm 0.18 \\
\text { strain }^{*}\end{array}$ & $0.30 \pm 0.05$ \\
\hline frontal ctx & NA/DA & DOPAC/DA & HVA/DA & $\begin{array}{l}\text { (DOPAC + } \\
\text { HVA)/DA }\end{array}$ & 5HIAA/5HT & 5HT/TRT & & \\
\hline Wistar & $6.40 \pm 1.77$ & $1.13 \pm 0.45$ & $0.57 \pm 0.20$ & $1.70 \pm 0.51$ & $1.68 \pm 0.40$ & $0.77 \pm 0.11$ & & \\
\hline WAG/Rij & $9.61 \pm 4.66$ & $2.29 \pm 1.76$ & $0.89 \pm 0.59$ & $3.18 \pm 2.35$ & $2.65 \pm 0.80$ & $0.81 \pm 0.24$ & & \\
\hline Wistar-AGS & $5.53 \pm 2.85$ & $1.27 \pm 0.58$ & $0.33 \pm 0.08$ & $1.59 \pm 0.64$ & $1.84 \pm 0.74$ & $0.80 \pm 0.08$ & & \\
\hline WAG/Rij-AGS & $5.98 \pm 1.33$ & $1.29 \pm 0.48$ & $0.39 \pm 0.08$ & $1.68 \pm 0.50$ & $1.99 \pm 0.74$ & $0.82 \pm 0.06$ & & \\
\hline parietal ctx & strain $^{*}$ & strain $^{*}$ & strain $^{*}$ & $\operatorname{strain}^{*}$ & & & & \\
\hline Wistar & $11.03 \pm 4.19$ & $0.56 \pm 0.19$ & $0.62 \pm 0.29$ & $1.18 \pm 0.44$ & $2.75 \pm 0.81$ & $0.12 \pm 0.07$ & & \\
\hline WAG/Rij & $12.04 \pm 5.87$ & $0.52 \pm 0.26$ & $1.23 \pm 0.71$ & $1.75 \pm 0.86$ & $4.40 \pm 1.10$ & $0.12 \pm 0.09$ & & \\
\hline Wistar-AGS & $10.41 \pm 5.53$ & $0.37 \pm 0.08$ & $1.92 \pm 1.23$ & $2.29 \pm 1.28$ & $3.55 \pm 1.07$ & $0.13 \pm 0.08$ & & \\
\hline $\begin{array}{c}\text { WAG/Rij-AGS } \\
\text { amygdala }\end{array}$ & $15.80 \pm 7.85$ & $0.26 \pm 0.10$ & $2.51 \pm 2.12$ & $\begin{array}{c}2.77 \pm 2.19 \\
\text { strain }^{*}\end{array}$ & $6.21 \pm 2.90$ & $0.08 \pm 0.04$ & & \\
\hline Wistar & $2.72 \pm 0.70$ & $0.38 \pm 0.17$ & $0.26 \pm 0.11$ & $0.58 \pm 0.30$ & $1.39 \pm 0.36$ & $0.93 \pm 0.16$ & & \\
\hline WAG/Rij & $4.94 \pm 1.01$ & $0.37 \pm 0.12$ & $0.47 \pm 0.13$ & $0.67 \pm 0.29$ & $1.20 \pm 0.20$ & $1.02 \pm 0.15$ & & \\
\hline Wistar-AGS & $2.99 \pm 1.45$ & $0.40 \pm 0.24$ & $0.44 \pm 0.19$ & $0.85 \pm 0.42$ & $1.22 \pm 0.22$ & $0.90 \pm 0.16$ & & \\
\hline WAG/Rij-AGS & $\begin{array}{c}2.86 \pm 0.40 \\
\text { strain }^{*}\end{array}$ & $0.16 \pm 0.06$ & $0.23 \pm 0.05$ & $0.38 \pm 0.10$ & $1.29 \pm 0.15$ & $0.90 \pm 0.11$ & & \\
\hline
\end{tabular}


(b)

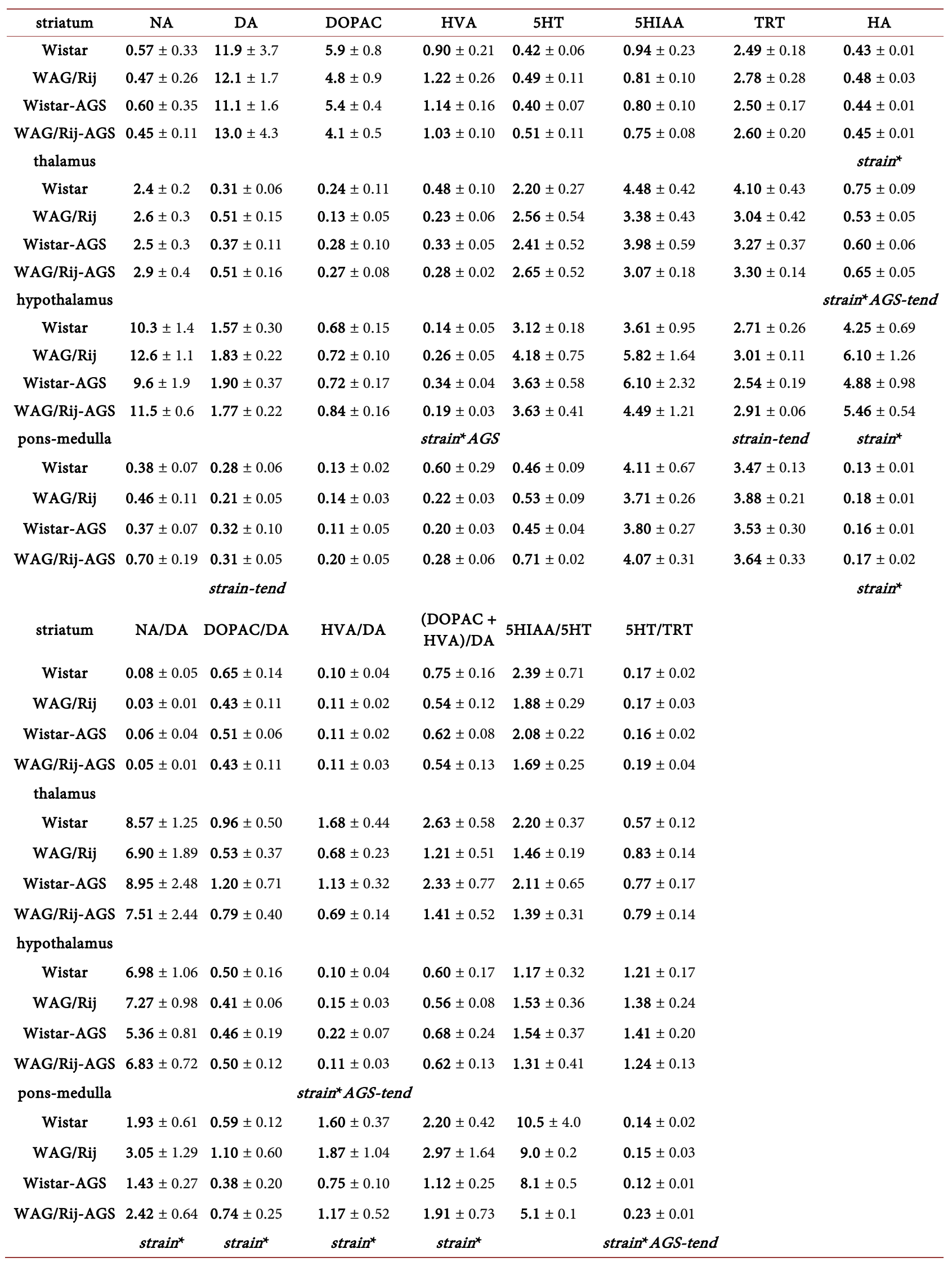


Thalamus: No significant strain difference was seen in this structure. HA tissue level tended for $\mathrm{AGS}^{*}$ strain interaction $\{\mathrm{F}(1,19)=4.10, \mathrm{p}=0.06\}$.

Pons-medulla: The tissue DA level tended to be lower $(\mathrm{p}=0.08)$, whereas that of TRT was higher in WAG/Rij rats (strain effect, $\{F(1,19)=8.00, p<0.01\}$ ). All metabolic ratios of DA were elevated, or tended to be elevated in WAG/Rij groups as compared to Wistar rats $(\{\mathrm{F}(1,19)=6.00, \mathrm{p}<0.05\},\{\mathrm{F}(1,19)=9.06, \mathrm{p}$ $<0.05\}$, and $\{\mathrm{F}(1,19)=8.45, \mathrm{p}<0.05\}$ for DOPAC/DA, HVA/DA and (DOPAC + HVA)/DA respectively\}, $\mathrm{p}=0.08$ for NA/DA). The rats with mixed type of epilepsy types had a higher synthesis rate of 5HT, as seen in the 5HT/TRT ratio $\left(\mathrm{AGS}^{*}\right.$ strain interaction $(\{\mathrm{F}(1,19)=6.65, \mathrm{p}<0.05\}$, all $\mathrm{p}<0.01$ in the post-hoc comparison of WAG/Rij-AGS to the other rat groups).

\subsection{Regional Interactions of the Aminergic Systems}

Those variables which did not differ (nor showed any tendency to differ) between AGS-prone and AGS-resistant groups of the same strain, were pooled together and subjected to a further inter-individual correlative analysis.

The relationships between the metabolite/precursor and the neurotransmitter were evident in many brain regions. These kinds of correlations are regarded as "an internal validation" of the method [8] and reflect the natural linkage between the compounds, usually via one or two enzymatic steps. These correlations are not shown here.

Correlations between the brain aminergic systems are given in Figure 1(a) and Figure 1(b). Briefly, these kinds of correlations were present in each studied region, with the exception of frontal cortex. The amount of inter-correlated aminergic indices differed between the brain regions and between the strains. One consistent phenomenon seen in both strains (underlined Rs, Figure 1), was the large amount of cortical correlations: positive between the tissue amounts of monoamines (i.e. NA, DA, 5HT) (parietal cortex in Figure 1(a)), and negative between the tissue $5 \mathrm{HT}$ indices and the DA turnover ratios (parietal cortex and amygdala in Figure 1(a)). Central brain regions demonstrated fewer common correlations for the two strains studied: striatum, thalamus, hypothalamus each displayed only 1 pair of correlated variables (5HT to the HVA/DA ratio, NA to the 5HIAA/5HT ratio, DA to 5HIAA respectively); pons-medulla displayed no similar links between the brain aminergic systems.

A strain difference appeared also in the patterns of regional correlations between NA, DA, HA and 5HT-ergic systems. Wistar rats had a higher (than WAG/Rij mates) number of correlated values within amygdala (27 and 15 out of 98 possible correlations for Wistar and WAG/Rij rats, respectively; $\mathrm{p}<0.02$ according to Fisher exact $2 \times 2$ test). WAG/Rij rats had a significantly higher number of correlated values within striatum (28 and 15 out of 98 possible correlations for WAG/Rij and Wistar strains, respectively; $\mathrm{p}<0.01$ according to Fisher exact $2 \times 2$ test), and pons-medulla (20 and 12 out of 98 possible correlations for WAG/Rij and Wistar respectively; $\mathrm{p}<0.05$ according to Fisher exact $2 \times 2$ test). 


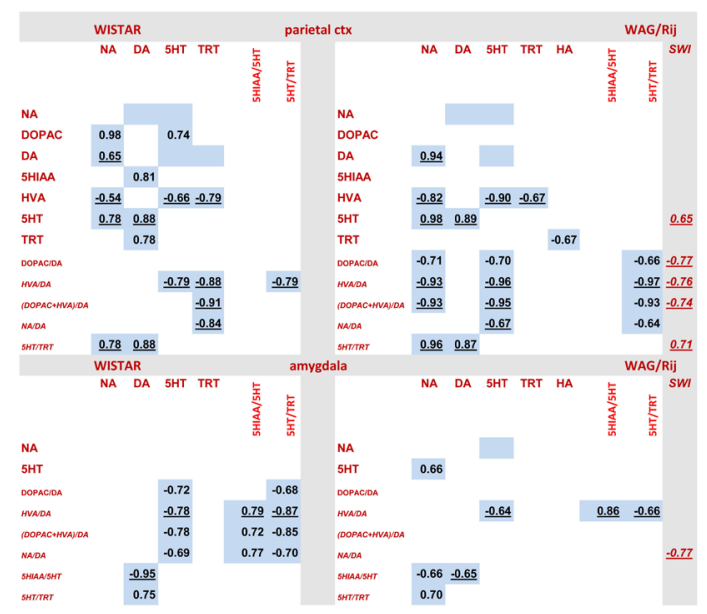

(a)

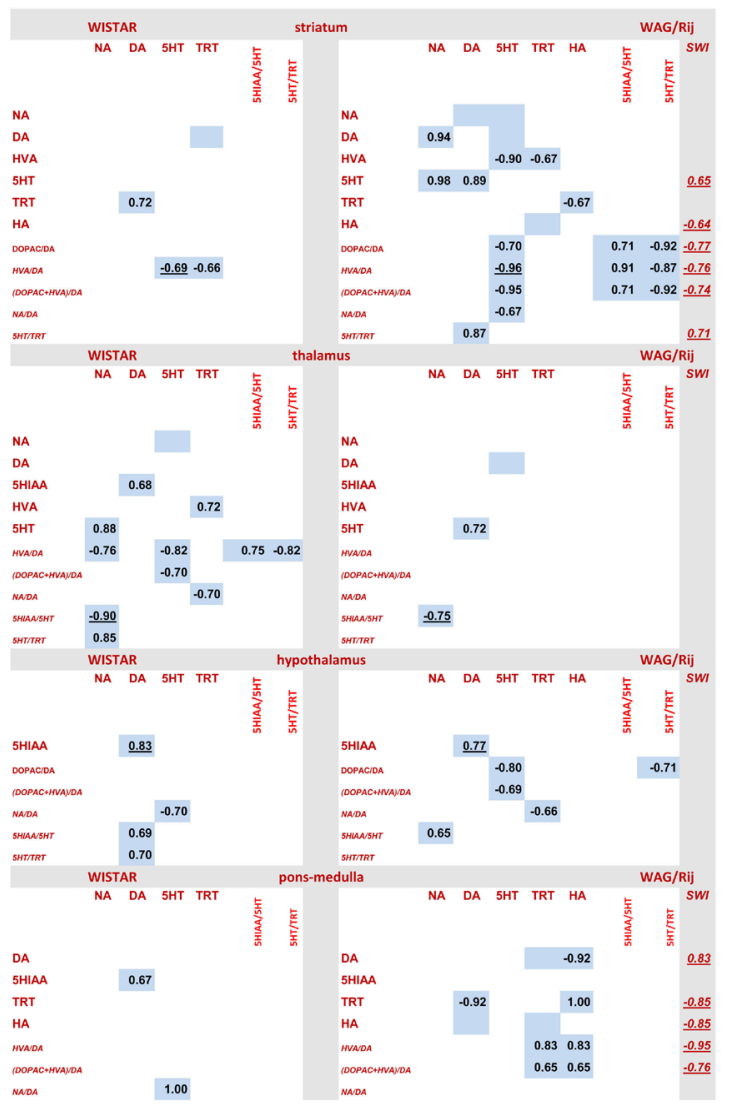

(b)

Figure 1. Correlative analysis of tissue contents for brain amines and their metabolic ratios within cortical (a) and subcortical (b) brain regions. Significant correlations (Spearman method of rank order correlation, Rs) BETWEEN noradrenergic (NA), dopaminergic (DA), serotonergic (5-HT) and histaminergic (HA) systems are given for each single brain regions. Nonsignificant values of Rs are omitted. Empty highlighted cells mark the places of symmetrical Rs values for the same correlations (i.e. the correlation of NA to $5 \mathrm{HT}$ is the same as the correlation $5 \mathrm{HT}$ to NA). Similar correlations seen in both strains are underlined. Rs for correlations of the individual indices of epileptic activity (SWI, only for WAG/Rij rats) and the regional aminergic variables are given in the right highlighted column, in underlined italic, nonsignificant Rs omitted. 
The severity of absence epilepsy in WAG/Rij rats (scored as SWI) correlated with a number of regional tissue aminergic indices, as reported before [11] [12] [13]. The relevant coefficients are given in Figure 1(a) and Figure 1(b) in italics. As can be seen, SWI was associated with several regional parameters of DA-ergic or 5 HTergic neurotransmission. In those cases, correlations between aminergic values were often stronger (Rs coefficient values higher, see the middle part of Figure 1(a) and Figure 1(b)) than the correlations of these variables with SWI itself (Rs coefficient values lower, see the last column of Figure 1(a) and Figure 1(b)). This was observed for parietal cortex, striatum, pons-medulla regions (Figure 1(a) and Figure 1(b)). One of these triple correlations is illustrated in Figure 2(a) and Figure 2(b): in striatum, individual SWI correlated negatively with DA metabolism and positively with the $5 \mathrm{HT}$ synthetic ratio ( $\mathrm{Rs}=-0.7$ and +0.7 ; see Figure 2(a)), whereas the striatal correlation of 5HT/TRT to (DOPAC + HVA)/DA was negative and appeared to be stronger $(\mathrm{Rs}=-0.9$; see Figure 2(b)).

Only WAG/Rij rats demonstrated correlations between tissue HA and other systems, mainly within the pons-medulla regions (Figure 1(b)). The correlation between the tissue levels of HA and TRT in pons-medulla was the strongest (Rs $=1$ ) in WAG/Rij rats, and was not present in Wistar rats. This particular region

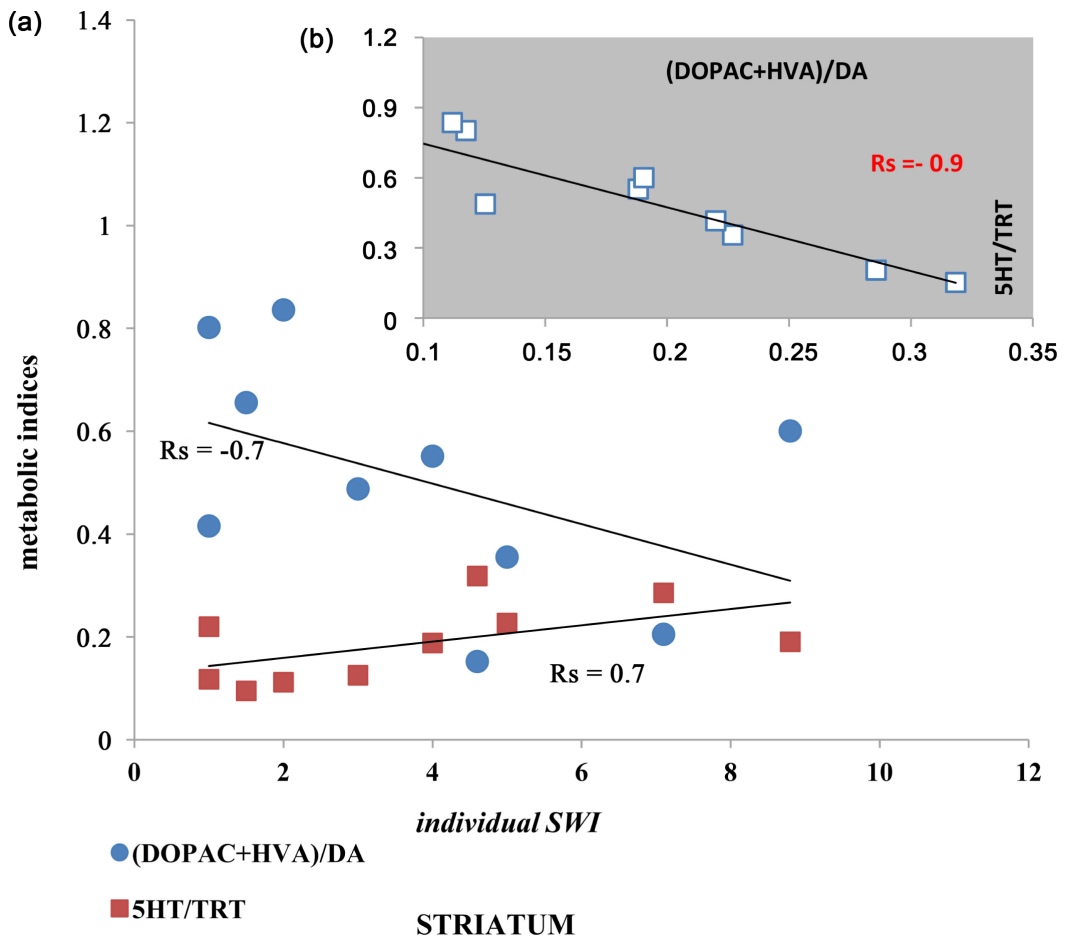

Figure 2. An example of regional multiple correlations. (a) Propensity towards absence epilepsy in WAG/Rij rats correlates negatively with the striatal dopamine turnover ratio, taken as (DOPAC + HVA)/DA, and positively with the serotonine sythetic ratio, taken as 5HT/TRT. The variables are depicted as blue circles and red squares, respectively; (b) The metabolic indices display a strong negative correlation, white squares. Rs are given beside the regression lines, all p's $<0.05$. 
displayed numerous correlations between SWI and aminergic indices (Figure 1(b), corresponded Rs are given in italic). In Wistar rats, tissue level of HA did not correlate with other aminergic indices.

\section{Discussion}

In order to address the widespread biochemical relationships of brain amines, we applied an inter-individual correlative analysis of the contents of tissue amines, their metabolites and metabolic ratios [10], which were measured within eight cerebral regions of normal and epileptic rat brains. The results obtained confirm and extend the findings of other authors [8]: brain aminergic systems interact in terms of correlated metabolic rates, in distal (cortex, striatum, thalamus) and central (pons-medulla, hypothalamus) cerebral regions (Figure 1(a) and Figure 1(b)). One phenomenon, seen in both strains (underlined Rs, Figure 1 ), was the large amount of positive correlations between the tissue amounts of monoamines (i.e. NA, DA, 5HT) assayed in the parietal cortex, as well as the negative correlations of tissue 5HT and the HVA/DA ratio in parietal cortex and amygdala (Figure 1(a)). This observation confirms the results reported by others [7] [8] [9] in Wistar rats. Presumably, it points to the general nature of these cortical interdependencies: tissue amount and turnover of biogenic amines at distal sites might have common regulatory mechanisms [9].

The metabolic rate of a monoamine has a greater functional significance than simply assaying the tissue concentration of the neurotransmitter itself [26] [27], and varies across brain regions [26] [34] [35] [36]. In fact, the components of the brain aminergic systems are targeted in the treatment of various neuropsychiatric and neurological conditions [3] [4]. Our results depict the "aminergic landscape" of normal rat brain and brain with recurrent spontaneous AbS. Clear functional correlations between striatal metabolic ratios of dopamine and serotonin were specific for absence epilepsy (Figure 1(a) and Figure 1(b)). If one considers the total amount of correlations (within and between aminergic systems), then the WAG/Rij rats displayed a significantly higher number of correlations within subcortical structures (striatum and pons-medulla) than Wistar rats (see Results). This highlights the tight connectivity between the DA and 5HTergic systems, which is specific for the AbS epileptic brain. For example, it is possible to assume that drugs targeting the 5HT-ergic system would have a lower threshold for DA-ergic side effects in epileptic brains. Similarly, the strongly coupled DA-ergic and 5HT-ergic metabolism might represent a deficiency in the vesicular monoamine transporter (VMAT2), which is present in both DA and 5HT neurons. Taking into account the fact that the actions of the biogenic amines are mainly terminated outside of the cells, i.e. within the synaptic clefts, if there was some deficiency in the transportation of stored compounds to the presynaptic membrane, this would account for these metabolic correlations. There are no data available on the functioning of VMAT1, 2 in patients with childhood absence epilepsy or in brains of animals with absence-type epilepsy. Interestingly, 
vesicular transporters have been associated with temporal lobe epilepsy [37]. Further investigations will be needed to clarify the role of this transporter in absence epilepsy.

It is evident that the correlations between striatal HVA/DA, DOPAC/DA, (DOPAC + HVA)/DA to 5HIAA/5HT, 5HT/TRT were stronger than those to SWI (see Figure 1(a) and Figure 1(b); Figure 2(a) and Figure 2(b)). This might mean that AbS aggravation is a secondary effect to the functional coupling of the DA-ergic and 5-HRergic systems.

One remarkable finding was that only in absence epileptic rats was the tissue HA level coupled to the tissue DA content and DA turnover indices in several brain regions (Figure 1(b)). For example, in pons-medulla of WAG/Rij rats, there were prominent positive correlations between the HA tissue content and the DA turnover indices (as well as a negative correlation between the tissue levels of HA and DA). These correlations were not present in Wistar rats (Figure $1(b)$ ). The aminergic parameters in WAG/Rij rat brain were all further correlated to the severity of absence epilepsy (as measured by the SWI) (Figure 1(b)). This illustrates a new feature of HA's actions, i.e. orchestrating DA-ergic transmission or metabolism in pons-medulla of WAG/Rij rats. There are reports in the literature that the HA-ergic system is activated if brain monoaminergic tone is low, as in the cases of Parkinson disease and in normally hibernating animals [38] [39]. A major increase in medullar HA content was detected in substantia nigra of human Parkinsonian brains [38]. The authors reported an increased density and thickness of histaminergic fibers, as well as enlarged HA-containing varicosities. This increase in histaminergic innervation has been proposed to compensate for the deficiency of DA [38]. In hibernating animals, monoaminergic activity is maintained at a very low level, in contrast to the clearly elevated HA turnover [38]. It has been hypothesized that histamine actively regulates the low activity of other transmitters during the hibernation state [40]. Modulation of firing of DA-ergic neurons has been reported as a key element in the functional interactions between histamine and other monoamines [40] with substantia nigra being proposed as a part of a putative "inhibitory control loop" for absence seizures [41] [42]. The functional significance of this HA-DA interaction within the pons-medulla region for the control of AbS remains to be elucidated.

The clearest correlation was found between tissue HA and TRT within ponsmedulla of WAG/Rij rats. This was somewhat unexpected and could not we find any explanation in the published literature. TRT has many functions; it is a dietary-supplied $\alpha$-amino acid that is used in the biosynthesis of proteins as well as a precursor of 5HT, melatonin (via 5HT) and niacin (vitamin B3, via kynurenine and quinolinic acids). Further experiments will be needed to clarify the mechanism and functional significance on the TRT-HA linkage.

It is notable that all groups of epileptic rats had elevated NA concentrations within the frontal cortex and amygdala (Table 1(a)). Interestingly, this elevated tissue NA did not correlate with SWI (Figure 1(a) and Figure 1(b)). Since an acute reduction of the noradrenergic neurotransmission was linked to an en- 
hancement of SWDs. In the WAG/Rij [43] as well as in the GAERS model [44] [45], it is possible to speculate that NA might be also poorly released and metabolized in these rats. Another possibility would be that there is an enhanced activity of the NA transporter, which can also take up DA from the synaptic cleft. This would explain both the elevated NA levels, unrelated to SWI, and the DA-ergic deficiency in cortical regions.

The study also allowed us to examine the putative interaction of convulsive and non-convulsive seizures on the level of brain amines. Nonetheless it appeared that this interaction effect was rather insignificant (Table 1(a) and Table 1(b); Results). AGS (convulsive seizures) are usually considered as more damaging to the brain than AbS (non-convulsive seizures). Nonetheless, we found that mild but recurrent absence paroxysms have a greater impact on the brain aminergic system than a single audiogenic convulsion, or a genetic predisposition to AGS.

The widespread DA-ergic deficiency parallels the AbS aggravation in WAG/Rij rats. Therefore, the effects of DA-ergic drugs also seem to depend on the propensity towards absence seizures. For example, undesirable motor side effects of psychostimulators or other DAergic drugs would be more prominent in individuals with severe AbS.

The unusual interaction pattern of the DA-ergic and 5HT-ergic systems may contribute to the depressive-like behavioral traits seen in WAG/Rij rats [46] [47].

The interaction pattern seen in the brain HA and DA-ergic systems within the pons-medulla of WAG/Rij rats resembles that encountered in the parkinsonian brain, perhaps signifying that there are common steps in pathogenesis of parkinsonian tremor and petit mal (nowadays called as childhood absence epilepsy) [5]. Interestingly in WAG/Rij rats, as well as in another genetic model of absence epilepsy, i.e. GAERS rats, DA-ergic deficiency appears prior to the first manifestation of AbS [48] [49] [50].

To conclude, by applying an inter-individual correlative approach, it was possible to reveal the specific "cerebral aminergic landscape" of absence epilepsy. In the absence epileptic brain, the regions of striatum and pons-medulla displayed a high grade of connectivity between brain biogenic amines, seen as a large number of correlations between DA, 5HT and HA-ergic indices. In the pons-medulla of WAG/Rij rats, there was evidence for HA control over DA turnover.

\section{Conflicts of Interest}

The authors declare no conflicts of interest regarding the publication of this paper.

\section{References}

[1] Bozzi, Y. and Borrelli, E. (2013) The Role of Dopamine Signaling in Epileptogenesis. 
Frontiers in Cellular Neuroscience, 7, 157. https://doi.org/10.3389/fncel.2013.00157

[2] Benes, F.M. (2000) Emerging Principles of Altered Neural Circuitry in Schizophrenia. Brain Research Reviews, 31, 251-269. https://doi.org/10.1016/S0165-0173(99)00041-7

[3] Di Giovanni, G., Svob Strac, D., Sole, M., Unzeta, M., Tipton, K.F., Mück-Šeler, D., Bolea, I., Della Corte, L., Nikolac Perkovic, M., Pivac, N., Smolders, I.J., Stasiak, A., Fogel, W.A. and De Deurwaerdère, P. (2016) Monoaminergic and Histaminergic Strategies and Treatments in Brain Diseases. Frontiers in Neuroscience, 10, 541. https://doi.org/10.3389/fnins.2016.00541

[4] Svob Strac, D., Pivac, N., Smolders, I.J., Fogel, W.A., De Deurwaerdere, P. and Di Giovanni, G. (2016) Monoaminergic Mechanisms in Epilepsy May Offer Innovative Therapeutic Opportunity for Monoaminergic Multi-Target Drugs. Frontiers in Neuroscience, 10, 492. https://doi.org/10.3389/fnins.2016.00492

[5] Buzsáki, G., Smith, A., Berger, S., Fisher, L.J. and Gage, F.H. (1990) Petit Mal Epilepsy and Parkinsonian Tremor: Hypothesis of a Common Pacemaker. Neuroscience, 36, 1-14. https://doi.org/10.1016/0306-4522(90)90345-5

[6] Starr, M.S. (1996) The Role of Dopamine in Epilepsy. Synapse, 22, 159-194. https://doi.org/10.1002/(SICI)1098-2396(199602)22:2<159::AID-SYN8>3.0.CO;2-C

[7] Fitoussi, A., Renault, P., Le Moine, C., Coutureau, E., Cador, M. and Dellu-Hagedorn, F. (2018) Inter-Individual Differences in Decision-Making, Flexible and Goal-Directed Behaviors: Novel Insights within the Prefronto-Striatal Networks. Brain Structure and Function, 223, 897-912. https://doi.org/10.1007/s00429-017-1530-Z

[8] Dellu-Hagedorn, F., Rivalan, M., Fitoussi, A. and De Deurwaerdère, P. (2018) Inter-Individual Differences in the Impulsive/Compulsive Dimension: Deciphering Related Dopaminergic and Serotonergic Metabolisms at Rest. Philosophical Transactions of the Royal Society of London. Series B, Biological Sciences, 373. https://doi.org/10.1098/rstb.2017.0154

[9] Dellu-Hagedorn, F., Fitoussi, A. and De Deurwaerdère, P.J. (2017) Correlative Analysis of Dopaminergic and Serotonergic Metabolism across the Brain to Study Monoaminergic Function and Interaction. Journal of Neuroscience Methods, 280, 54-63. https://doi.org/10.1016/j.jneumeth.2017.01.020

[10] Avakian, R.M. (1976) Effect of Striatectomy on the Course of Corazole Seizures in Rats. Bulletin of Experimental Biology and Medicine, 81, 316-319. https://doi.org/10.1007/BF00804923

[11] Midzyanovskaya, I.S. (2006) Absence and Mixed Forms of Epilepsy in WAG/Rij Rats [Electronic Resource]: Characteristics and Brain Aminergic Modulations. https://webdoc.ubn.ru.nl/mono/m/midzyanovskaya_i/abseanmif.pdf

[12] Midzyanovskaya, I.S., Kuznetsova, G.D., van Luijtelaar, E.L., van Rijn, C.M., Tuomisto, L. and Macdonald, E. (2006) The Brain 5HTergic Response to an Acute Sound Stress in Rats with Generalized (Absence and Audiogenic) Epilepsy. Brain Research Bulletin, 69, 631-638. https://doi.org/10.1016/j.brainresbull.2006.03.008

[13] Midzyanovskaya, I.S., Kuznetsova, G.D. and Tuomisto, L. (2002) Brain Histamine in the WAG/Rij Rat, an Animal Model of Absence Epilepsy. Inflammation Research, 51, S49-S50.

[14] Deransart, C., Lê-Pham, B.T., Hirsch, E., Marescaux, C. and Depaulis, A. (2001) Inhibition of the Substantia Nigra Suppresses Absences and Clonic Seizures in Audiogenic Rats, but Not Tonic Seizures: Evidence for Seizure Specificity of the Nigral Control. Neuroscience, 105, 203-211. https://doi.org/10.1016/S0306-4522(01)00165-8 
[15] Tuomisto, L. and Tacke, U. (1986) Is Histamine an Anticonvulsive Inhibitory Transmitter? Neuropharmacology, 25, 955-958. https://doi.org/10.1016/0028-3908(86)90029-8

[16] Jobe, P.C. and Browning, R.A. (2005) The Serotonergic and Noradrenergic Effects of Antidepressant Drugs Are Anticonvulsant, Not Proconvulsant. Epilepsy and Behavior, 7, 602-619. https://doi.org/10.1016/j.yebeh.2005.07.014

[17] Weinshenker, D. and Szot, P. (2002) The Role of Catecholamines in Seizure Susceptibility: New Results Using Genetically Engineered Mice. Pharmacology \& Therapeutics, 94, 213-233. https://doi.org/10.1016/S0163-7258(02)00218-8

[18] Morales-Villagrán, A., López-Pérez, S., Medina-Ceja, L. and Tapia, R. (1999) Cortical Catecholamine Changes and Seizures Induced by 4-Aminopyridine in Awake Rats, Studied with a Dual Microdialysis-Electrical Recording Technique. Neuroscience Letters, 275, 133-136. https://doi.org/10.1016/S0304-3940(99)00759-4

[19] Tuomisto, L., Tacke, U. and Willman, A. (1987) Inhibition of Sound-Induced Convulsions by Metoprine in the Audiogenic Seizure Susceptible Rat. Agents and Actions, 20, 252-254. https://doi.org/10.1007/BF02074683

[20] Yokoyama, H., Onodera, K., Mayema, K., Yanai, K., Iinuma, K., Tuomisto, L. and Watanabe, T. (1992) Histamine Levels and Clonic Convulsions of Electrically-Induced Seizure in Mice: The Effects of Alfa-Fluoromethylhistidine and Metoprine. Naunyn-Schmiedeberg's Archives of Pharmacology, 346, 40-45. https://doi.org/10.1007/BF00167568

[21] Coenen, A.M. and Van Luijtelaar, E.L. (2003) Genetic Animal Models for Absence Epilepsy: A Review of the WAG/Rij Strain of Rats. Behavior Genetics, 33, 635-655. https://doi.org/10.1023/A:1026179013847

[22] van Luijtelaar, E.L. and Coenen, A.M. (1986) Two Types of Electrocortical Paroxysms in an Inbred Strain of Rats. Neuroscience Letters, 70, 393-397. https://doi.org/10.1016/0304-3940(86)90586-0

[23] Schridde, U. and van Luijtelaar, G. (2004) The Influence of Strain and Housing on Two Types of Spike-Wave Discharges in Rats. Genes, Brain and Behavior, 3, 1-7. https://doi.org/10.1111/j.1601-1848.2004.00034.x

[24] Kuznetsova, G.D. (1998) Audiogenic Seizures in Different Rat Strains. Zh Vysh Nervn Deyat., 48, 143-152. (In Russian)

[25] Midzyanovskaya, I.S., Kuznetsova, G.D., Vinogradova, L.V., Shatskova, A.B., Coenen, A.M. and van Luijtelaar, G. (2004) Mixed Forms of Epilepsy in a Subpopulation of WAG/Rij Rats. Epilepsy and Behavior, 5, 655-661. https://doi.org/10.1016/j.yebeh.2004.06.021

[26] Commissiong, J.W. (1985) Monoamine Metabolites: Their Relationship and Lack of Relationship to Monoaminergic Neuronal Activity. Biochemical Pharmacology, 34, 1127-1131. https://doi.org/10.1016/0006-2952(85)90484-8

[27] Eisenhofer, G., Kopin, I.J. and Goldstein, D.S. (2004) Catecholamine Metabolism: A Contemporary View with Implications for Physiology and Medicine. Pharmacological Reviews, 56, 331-349. https://doi.org/10.1124/pr.56.3.1

[28] Glowinski, J. (1975) Properties and Functions of Intraneuronal Monoamine Compartments in Central Aminergic Neurons. In: Iversen, L.L., Iversen, S.D. and Snyder, S.H., Eds., Biochemistry of Biogenic Amines. Handbook of Psychopharmacology (Section I: Basic Neuropharmacology), Springer, Boston, MA, 139-167. https://doi.org/10.1007/978-1-4684-3171-1_3

[29] Green, R. and Grahame-Smith, D.G. (1975) 5-Hydroxytryptamine and Other In- 
doles Inthe Central Nervous System. In: Iversen, L.L., Iversen, S.D. and Snyder, S.H., Eds., Biochemistry of Biogenic Amines. Handbook of Psychopharmacology (Section I: Basic Neuropharmacology, Springer, Boston, MA, 169-245. https://doi.org/10.1007/978-1-4684-3171-1_4

[30] Glowinski, J. and Iversen, L.L. (1966) Regional Studies of Catecholamines in the Rat Brain. I. The Disposition of [3H]Norepinephrine, [3H]Dopamine and [3H]Dopa in Various Regions of the Brain. Journal of Neurochemistry, 13, 655-669. https://doi.org/10.1111/j.1471-4159.1966.tb09873.x

[31] Midzyanovskaya, I.S., Shatskova, A.B., Sarkisova, K.Y., van Luijtelaar, G., Tuomisto, L. and Kuznetsova, G.D. (2005) Convulsive and Nonconvulsive Epilepsy in Rats: Effects on Behavioral Response to Novelty Stress. Epilepsy and Behavior, 6, 543-551. https://doi.org/10.1016/j.yebeh.2005.03.005

[32] Mefford, I.N. (1981) Application of High Performance Liquid Chromatography with Electrochemical Detection to Neurochemical Analysis: Measurement of Catecholamines, Serotonin and Metabolites in Rat Brain. Journal of Neuroscience Methods, 3, 207-224. https://doi.org/10.1016/0165-0270(81)90056-X

[33] Yamatodani, A., Fukuda, H., Iwaeda, T., Wada, H., Wada, H. and Watanabe, T. (1985) High-Performance Liquid Chromatographic Determination of Plasma and Brain Histamine without Previous Purification of Biological Samples: Cation-Exchange Chromatography Coupled with Post-Column Derivatization Fluorometry. Journal of Chromatography B, 344, 115-123. https://doi.org/10.1016/S0378-4347(00)82012-5

[34] Fitoussi, A., Dellu-Hagedorn, F. and De Deurwaerdère, P. (2013) Monoamines Tissue Content Analysis Reveals Restricted and Site-Specific Correlations in Brain Regions Involved in Cognition. Neuroscience, 255, 233-245. https://doi.org/10.1016/j.neuroscience.2013.09.059

[35] Bannon, M.J., Bunney, E.B. and Roth, R.H. (1981) Mesocortical Dopamine Neurons: Rapid Transmitter Turnover Compared to Other Brain Catecholamine Systems. Brain Research, 218, 376-382.

[36] Shannon, N.J., Gunnet, J.W. and Moore, K.E. (1986) A Comparison of Biochemical Indices of 5-Hydroxytryptaminergic Neuronal Activity Following Electrical Stimulation of the Dorsal Raphe Nucleus. Journal of Neurochemistry, 47, 958-965. https://doi.org/10.1111/j.1471-4159.1986.tb00704.x

[37] Van Liefferinge, J., Massie, A., Portelli, J., Di Giovanni, G. and Smolders, I. (2013) Are Vesicular Neurotransmitter Transporters Potential Treatment Targets for Temporal Lobe Epilepsy? Frontiers in Cellular Neuroscience, 7, 139. https://doi.org/10.3389/fncel.2013.00139

[38] Anichtchik, O.V., Rinne, J.O., Kalimo, H. and Panula, P. (2000) An Altered Histaminergic Innervation of the Substantia Nigra in Parkinson's Disease. Experimental Neurology, 163, 20-30. https://doi.org/10.1006/exnr.2000.7362

[39] Panula, P., Karlstedt, K., Sallmen, T., Peitsaro, N., Kaslin, J., Michelsen, K.A., Anichtchik, O., Kukko-Lukjanov, T. and Lintunen, M. (2000) The Histaminergic System in the Brain: Structural Characteristics and Changes in Hibernation. Journal of Chemical Neuroanatomy, 18, 65-74. https://doi.org/10.1016/S0891-0618(99)00052-6

[40] Flik, G., Folgering, J.H., Cremers, T.I., Westerink, B.H. and Dremencov, E. (2015) Interaction between Brain Histamine and Serotonin, Norepinephrine, and Dopamine Systems: In Vivo Microdialysis and Electrophysiology Study. Journal of Molecular Neuroscience, 56, 320-328. https://doi.org/10.1007/s12031-015-0536-3 
[41] Nehlig, A., Vergnes, M., Boyet, S. and Marescaux, C. (1998) Local Cerebral Glucose Utilization in Adult and Immature GAERS. Epilepsy Research, 32, 206-212. https://doi.org/10.1016/S0920-1211(98)00052-7

[42] Deransart, C. and Depaulis, A. (2002) The Control of Seizures by the Basal Ganglia? A Review of Experimental Data. Epileptic Disorders, 4, S61-S72.

[43] Sitnikova, E. and van Luijtelaar, G. (2005) Reduction of Adrenergic Neurotransmission with Clonidine Aggravates Spike-Wave Seizures and Alters Activity in the Cortex and the Thalamus in WAG/Rij Rats. Brain Research Bulletin, 64, 533-540. https://doi.org/10.1016/j.brainresbull.2004.11.004

[44] Lannes, B., Vergnes, M., Marescaux, C., Depaulis, A., Micheletti, G., Warter, J.M. and Kempf, E. (1991) Lesions of Noradrenergic Neurons in Rats with Spontaneous Generalized Non-Convulsive Epilepsy. Epilepsy Research, 9, 79-85.

https://doi.org/10.1016/0920-1211(91)90016-9

[45] Micheletti, G., Warter, J.M., Marescaux, C., Depaulis, A., Tranchant, C., Rumbach, L. and Vergnes, M. (1987) Effects of Drugs Affecting Noradrenergic Neurotransmission in Rats with Spontaneous Petit Mal-Like Seizures. European Journal of Pharmacology, 135, 397-402. https://doi.org/10.1016/0014-2999(87)90690-X

[46] Sarkisova, K. and van Luijtelaar, G. (2011) The WAG/Rij Strain: A Genetic Animal Model of Absence Epilepsy with Comorbidity of Depression. Progress in Neuro-Psychopharmacology and Biological Psychiatry, 35, 854-876. https://doi.org/10.1016/j.pnpbp.2010.11.010

[47] Sarkisova, K.Y., Kulikov, M.A., Midzyanovskaya, I.S. and Folomkina, A.A. (2008) Dopamine-Dependent Nature of Depression-Like Behavior in WAG/Rij Rats with Genetic Absence Epilepsy. Neuroscience and Behavioral Physiology, 38, 119-128. https://doi.org/10.1007/s11055-008-0017-Z

[48] Birioukova, L.M., Sitnikova, E.Y., Kulikov, M.A. and Raevsky, V.V. (2016) Compensatory Changes in the Brain Dopaminergic System of WAG/Rij Rats Genetically Predisposed to Absence Epilepsy. Bulletin of Experimental Biology and Medicine, 161, 662-665. https://doi.org/10.1007/s10517-016-3480-5

[49] Cavarec, F., Krauss, P., Witkowski, T., Broisat, A., Ghezzi, C., De Gois, S., Giros, B., Depaulis, A. and Deransart, C. (2019) Early Reduced Dopaminergic Tone Mediated by D3 Receptor and Dopamine Transporter in Absence Epileptogenesis. Epilepsia, 60, 2128-2140. https://doi.org/10.1111/epi.16342

[50] Sitnikova, E., Egorova, T.N. and Raevski1̌, V.V. (2012) Reduction of the Number of Neurons in Substantia Nigra (Pars Compacta) Positively Correlates with a Reduction of Seizure Activity in WAG/Rij Rats. Zhurnal vyssher nervnor deiatelnosti imeni I P Pavlova, 62, 619-628. (In Russian) 